\title{
Distribución y abundancia de la gamba Haliporoides diomedeae (Crustacea: Decapoda: Penaeidae) frente a la costa central de Chile
}

\author{
Patricio M. Arana ${ }^{1}$, Mauricio Ahumada ${ }^{2}$ \& Aurora Guerrero ${ }^{2}$ \\ Departamento de Recursos Bentodemersales \\ Escuela de Ciencias del Mar, Pontificia Universidad Católica de Valparaíso \\ Casilla 1020, Valparaíso, Chile \\ E-mail: ${ }^{1}$ parana@ucv.cl; ${ }^{2}$ bentodem@ucv.cl
}

\begin{abstract}
RESUMEN: Con la finalidad de reunir antecedentes biológico-pesqueros, determinar la distribución y evaluar la biomasa vulnerable de la gamba (Haliporoides diomedeae), se prospectó con red de arrastre de fondo el talud continental frente a la costa central de Chile (32 $10^{\circ} \mathrm{S}$ a $34^{\circ} 42^{\prime} \mathrm{S}$ ), entre el 8 de agosto y el 16 de septiembre de 2002 . El diseño de muestreo fue bi-etápico, con transectas perpendiculares a la costa, separadas entre sí por $10 \mathrm{mn}$, y lances adicionales entre ellas para mejorar la precisión de los estimados. La captura de esta especie totalizó 11 ton. En cada lance se tomaron muestras, midiéndose un total de 50.713 ejemplares, con longitudes cefalotorácicas $\left(\mathrm{L}_{\mathrm{c}}\right)$ entre 12 y 72 $\mathrm{mm}$, con media de 30,6 mm y moda en $28 \mathrm{~mm}$. La talla de madurez física, se estableció en 32,5 $\mathrm{mm}$ de $\mathrm{L}_{\mathrm{c}}$ en machos y $36,5 \mathrm{~mm}$ de $\mathrm{L}_{\mathrm{c}}$ en hembras. Durante el estudio se realizó un total 265 lances de pesca entre 295 y $682 \mathrm{~m}$, registrándose 179 arrastres positivos de gamba, con los más altos rendimientos a profundidades mayores de $500 \mathrm{~m}$. Este recurso se distribuyó en dos conglomerados de abundancia de 620,5 y $539,2 \mathrm{~km}^{2}$ cada uno, separados por el cañón submarino de San Antonio. La biomasa vulnerable en el área de estudio se estimó entre 702 y 745 ton, de la cual 237,7 ton correspondió a ejemplares con longitudes sobre la talla de madurez física. De acuerdo a los resultados obtenidos, la gamba constituiría un recurso complementario a otros crustáceos explotados en forma tradicional en aguas menos profundas.
\end{abstract}

Palabras claves: gamba, Haliporoides diomedeae, distribución, distribuciones de tallas, abundancia, Chile.

\section{Distribution and abundance of red royal shrimp Haliporoides diomedeae (Crustacea: Decapoda: Penaeidae) off central coast of Chile}

\begin{abstract}
A survey over the continental slope off the central coast of Chile (32 $10^{\circ} \mathrm{S}$ a $34^{\circ} 42^{\prime}$ 'S), with a bottom trawl net, was conducted to gather fishing-biological antecedents and assess the red royal shrimp resource (Haliporoides diomedeae), between August 8th and September 16th, 2002. The sampling design was set in two steps, with transects perpendicular to the coast, separated by $10 \mathrm{~nm}$. Assessment hauls between them were performed to improve the estimates precision. The total catch of the species reached 11 ton. Samples were taken on each haul. Measurements were taken on 50.713 specimens, recording carapace lengths $\left(\mathrm{L}_{c}\right)$ between 12 and $72 \mathrm{~mm}$, with a mean of $30 \mathrm{~mm}$ and mode at 28 $\mathrm{mm}$. Length at physical maturity $\left(\mathrm{L}_{\mathrm{c}}\right)$, was established in $32,5 \mathrm{~mm}$ for males and $37,5 \mathrm{~mm}$ for females. During the study a total of 265 fishing hauls were taken between 295 and $682 \mathrm{~m}$, obtaining 179 positive trawls with red royal shrimps. The highest yields were obtained over $500 \mathrm{~m}$ depth. The resource was found to be distributed in two abundance aggregates of 620,5 and 539,2 $\mathrm{km}^{2}$ each, separated by the San Antonio Canyon. The vulnerable biomass determined in the study area was between 702 and 745 tons, of which 237,7 tons correspond to specimens with length over first physical maturity According to these results, the red royal shrimp is positioned as a complementary resource among other crustaceans traditionally exploited in shallower waters.
\end{abstract}

Key words: red royal shrimp, Haliporoides diomedeae, distribution, size composition, abundance, Chile. 


\section{INTRODUCCIÓN}

Durante el último trienio, la disminución en la disponibilidad de los crustáceos explotados en fondos marinos frente a la costa central de Chile ( $32^{\circ} 10^{\prime} \mathrm{S}$ a $37^{\circ} 00^{\prime} \mathrm{S}$ ) ha motivado a la autoridad a decretar vedas extractivas y/o la fijación de cuotas reducidas destinadas a preservar las poblaciones de los principales recursos extraídos como el camarón nailon (Heterocarpus reedi), langostino amarillo (Cervimunida johni) y langostino colorado (Pleuroncodes monodon) (Barbieri et al., 2000; Escuela de Ciencias del Mar, 2000a, 2000b; Canales et al., 2001; Arana et al., 2003). Dada esta situación, empresas partícipes de dichas pesquerías han debido cesar sus funciones o disminuirlas en forma notoria, lo cual ha motivado la reorientación de sus operaciones de pesca a caladeros situados al norte de esta área, los cuales están actualmente sometidos a una fuerte presión de pesca por parte de la flota (Guerrero et al., 2003). De acuerdo a ello, una alternativa a la explotación actual de crustáceos es diversificar la extracción hacia especies no explotadas o sometidas a bajos niveles de captura, como es el caso de la gamba (Haliporoides diomedeae Faxon, 1893).

Esta especie ha sido registrada desde el golfo de Panamá $\left(07^{\circ} 31^{\prime} \mathrm{N}\right)$ hasta los $42^{\circ} 30^{\prime} \mathrm{S}$, con registros en profundidades entre 300 y 1.866 m (Faxon, 1895; Pérez-Farfante, 1977; Leiva et al., 1997). En Chile, hasta ahora $H$. diomedeae ha sido escasamente estudiada dado que se distribuye en fondos de mayor profundidad a los utilizados regularmente por la flota industrial (Yáñez, 1974; Yáñez \& Barbieri, 1974; Noziglia \& Arana, 1976; Henríquez et al., 1981; Vélez et al., 1992; Leiva et al., 1997). La orientación de operaciones extractivas hacia este recurso requiere del respaldo de antecedentes técnicos, motivo por el cual los objetivos de este trabajo son la determinación de índices de abundancia relativa (CPUE y CPUA), biomasa vulnerable, estructura de tallas y caracterización de la fauna capturada en faenas de extracción de la gamba frente a la costa central de Chile.

\section{MATERIALES Y MÉTODOS}

El área de estudio comprendió los fondos marinos frente a la zona central de Chile, entre los $32^{\circ} 10^{\prime} \mathrm{S}$ y $34^{\circ} 42^{\prime} \mathrm{S}$. Las labores extractivas, con redes de arrastre de fondo, de $40 \mathrm{~mm}$ de tamaño de malla en el copo, se efectuaron entre 295 y 682 m de profundidad (Fig. 1).

Los datos se recopilaron simultáneamente a bordo de dos embarcaciones. De este modo, en una primera etapa se utilizó las naves "Crusoe I" (Loa 23,6 m) y "Elbe" (Loa 23,6 m), que posteriormente fueron reemplazadas por los pesqueros "Don Stefan" (Loa 23,6 m) y "Maorí" (Loa 23,6 m), todas ellas de Pesquera Quintero S.A.

El diseño de trabajo consideró un muestreo de carácter bietápico, que utilizó lances sistemáticos y de evaluación, que ha sido empleado en numerosos trabajos de evaluación directa (Escuela de Ciencias del Mar, 2000a; Canales et al., 2001; Arana et al., 2003). La primera etapa, de carácter sistemático, se orientó a identificar la cobertura espacial de la gamba, mientras que la segunda, consistió en aumentar la precisión de los estimados mediante el incremento del número de lances de pesca, en aquellas zonas en las cuales se había detectado previamente la presencia del recurso objetivo.

Los lances sistemáticos se distribuyeron en transectas perpendiculares a la costa, separadas entre sí por 10 millas náuticas. Estas se dispusieron desde el extremo norte del área de estudio (32¹0'S), planificándose seis lances en cada una de ellas, aproximadamente a 300, 400, 450, 500, 600 y 675 $\mathrm{m}$ de profundidad. En la segunda etapa, se efectuaron lances de evaluación, con tres lances promedio entre transectas consecutivas. Los lances sistemáticos tuvieron una duración de una hora, mientras que los de evaluación de tres horas. En ambos casos, el tiempo de arrastre correspondió al instante medido desde la finalización del calado hasta el inicio del virado de la red.

En cada lance de pesca, se registró la posición de calado y virado según lectura de GPS, profundidad de arrastre de acuerdo a lo indicado en el ecosonda y la captura por especie. Se contabilizó el número de cajas de captura de gamba, y mediante el peso promedio de cinco de ellas, se calculó la captura total por lance, en kg. Se separó una caja de pesca por lance, para determinar el sexo (Arana \& Cristi, 1971; Illanes \& Zúñiga, 1972), longitud cefalotorácica $(\mathrm{mm})$, longitud total $(\mathrm{mm})$ y peso individual.

La estructura de tallas se obtuvo mediante distribuciones de frecuencias, agrupadas en intervalos de $1 \mathrm{~mm}$, separadamente en machos y hembras. En cada caso se calculó la talla media y la moda. La proporción sexual se estableció de acuerdo al por- 


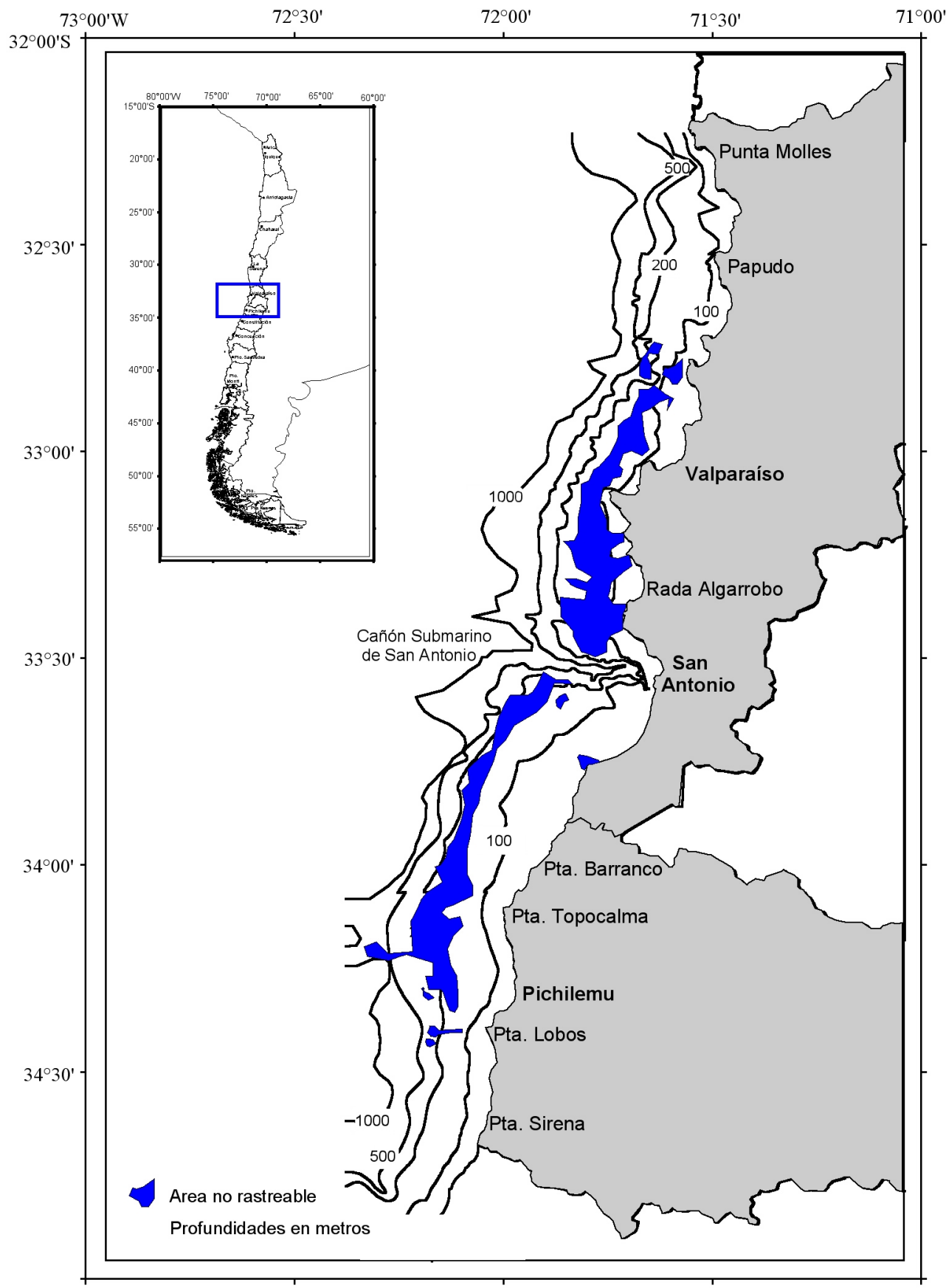

Figura 1. Zona de prospección durante el crucero de evaluación de la gamba, indicándose el área no rastreable. Figure 1. Surveyed zone during the assessment cruise of the red royal shrimp. Non trawlable area is indicated. 
centaje de machos en cada lance. Respecto de la relación longitud-peso, se empleó la función de poder tradicional, cuyos parámetros se obtuvieron del ajuste de mínimos cuadrados, previa linealización de la función mediante logaritmo natural (ln).

Para establecer el tipo de crecimiento relativo (alométrico-isométrico), se aplicó la prueba t de Student (Dixon \& Massey, 1957) mediante formulación de la dócima de hipótesis $\mathrm{H}_{0}: \mathrm{b}=3, \mathrm{H}_{1}: \mathrm{b} \bullet 3$. La igualdad de la relación talla-peso entre machos y hembras se probó haciendo uso del test F (1- $\alpha$; 2 , $\left.\mathrm{n}_{1}+\mathrm{n}_{2}-4\right)$ (Neter \& Wasserman, 1974), cuya expresión es:

$$
F^{*}=\frac{\frac{S S E_{M+H}-S S E_{T}}{2}}{\frac{S S E_{T}}{n_{1}+n_{2}-4}}
$$

donde:

$$
S S E=\sum\left\{\left(\ln \left(W_{L c i}\right)-\ln (a)-b \cdot \ln (L c)\right\}^{2}\right.
$$

siendo $S S E_{M+H}$ la suma del cuadrado de los errores de la regresión combinada de machos y hembras, y $S S E_{T}$ la suma del cuadrado de los errores en machos $\left(S S E_{M}\right)$ y en hembras $\left(S S E_{H}\right)$.

Siguiendo el enfoque utilizado en diversas especies de crustáceos por Gray \& Newcombe (1938), George \& Morgan (1979), Somerton (1980), Conan \& Comeau (1986) y Reigada et al. (1994), la estimación de la talla de madurez física se efectuó mediante el análisis de las relaciones morfométricas entre la longitud total $\left(\mathrm{L}_{\mathrm{t}}\right)$ y la longitud cefelotorácica $\left(\mathrm{L}_{\mathrm{c}}\right)$. Esto se realiza por cuanto la gamba corresponde a un camarón peneido, no portador de huevos entre sus pleópodos, lo que impide la determinación de la talla de primera madurez sexual (TMS) utilizando ojivas que representan el porcentaje de hembras maduras en cada rango de longitud, método empleado tradicionalmente en especie portadoras.

La longitud de madurez física se determinó en machos y hembras considerando el quiebre en la relación $\mathrm{L}_{\mathrm{c}}-\mathrm{L}_{\mathrm{t}}$. De acuerdo con lo señalado por Noziglia \& Arana (1976) en esta misma especie, a cierta talla se produce un cambio de pendiente en esa relación, la cual correspondería al momento en que alcanza la madurez física.

Con este fin, se utilizó el procedimiento iterativo desarrollado por Inostroza (1981), que corresponde a una modificación de la técnica propuesta anterior- mente por Quandt (1958). El método se fundamenta en el análisis de regresiones bifásicas, con el cual se determina el lugar geométrico de cambio entre dos fases al calcular la función de verosimilitud $(Q)$, que entrega en dicho punto un máximo. Este estimador se expresa de la siguiente manera:

$$
Q=-N \cdot(2 \cdot \pi)-n \cdot \ln \left(S_{1}\right)-(N-n) \cdot \ln \left(S_{2}\right)-\left(\frac{N}{2}\right)
$$

donde:

$N$ : Número total de observaciones (fase $1+$ fase 2)

$n$ : Número de observaciones utilizadas en el ajuste de la fase 1

$s_{1}$ : Desviación estándar asociada a la fase 1

$s_{2}$ : Desviación estándar asociada a la fase 2

Para aplicar este método, primeramente se agrupó las mediciones por rangos de un milímetro de $\mathrm{L}_{\mathrm{C}}$ y luego se calculó los valores medios de $\mathrm{L}_{\mathrm{T}}$ para cada marca de clase. Con esto se pretende detectar y corregir posibles errores en la medición de los ejemplares y eliminar la variabilidad inherente al desarrollo individual de los mismos. Una vez graficados los pares de valores, en escala natural o logarítmica, se analizó la serie de registros $\mathrm{L}_{\mathrm{C}}-\mathrm{L}_{\mathrm{T}}$ mediante el método iterativo antes indicado, para determinar cambios en la trayectoria de las curvas. Posteriormente, se ajustaron por mínimos cuadrado los valores correspondientes a cada fase que presenta igual tendencia y, finalmente, se determinó el punto de inflexión entre ellas.

Con docimacia de hipótesis se comprobó la diferencia entre los ajustes lineales de individuos maduros e inmaduros, contrastando la hipótesis nula $\mathrm{H}_{0}: \beta_{01}=\beta_{02}$ y $\beta_{11}=\beta_{12}$ con la hipótesis alternativa $\mathrm{H}_{1}$ : $\beta_{01} \bullet \beta_{02}$ o $\beta_{11} \bullet \beta_{12}$. Para ello se utilizó el estadístico $\mathrm{F}$, que fue contrastado con el valor de $\mathrm{F}$ de Fisher $\mathrm{F}\left(1-\alpha, 2, \mathrm{n}_{1}+\mathrm{n}_{2}-4\right)$ (Neter \& Wasserman, 1974), donde:

$$
F^{*}=\frac{\frac{S S E(R)-S S E(F)}{2}}{\frac{S S E(F)}{n_{1}+n_{2}-4}}
$$

siendo $\mathrm{SSE}(\mathrm{R})$ y $\mathrm{SSE}(\mathrm{F})$ la suma de cuadrados de error de los modelos reducidos (inmaduros o madu- 
ros) y combinado (maduros e inmaduros), respectivamente.

Para establecer la distribución espacial del recurso, se procedió a la georreferenciación de los lances de pesca con y sin captura. Con el fin de determinar los límites de cada caladero, se utilizaron los márgenes de los fondos no rastreables, los límites batimétricos de los lances con captura y la distancia entre lances con y sin captura. Para ello se recurrió a cartas del Servicio Hidrográfico y Oceanográfico de la Armada, previamente digitalizadas. La superficie de agregación del recurso se estimó con un planímetro digital.

El rendimiento de pesca se expresó en términos de captura por unidad de esfuerzo (CPUE), en kilos por hora de arrastre (kg/h.a.). Esto con el propósito de comparar este índice con estudios efectuados anteriormente y aportar con antecedentes para evaluar su extracción comercial.

Con el objeto de estimar la biomasa vulnerable del recurso, se empleó el método de evaluación directa de área barrida, que consiste en calcular la densidad de la especie objetivo, relacionando su captura con el área barrida por la red y extrapolándola a la totalidad del área de evaluación. De este modo, el principal supuesto en este procedimiento es que la densidad o abundancia estimada es proporcional a la abundancia presente en la totalidad del área prospectada (Alverson \& Pereyra, 1969; Troadec, 1980; Sparre \& Venema, 1995), considerando un coeficiente de capturabilidad equivalente a la unidad.

El área barrida por la red de arrastre de fondo $\left(\mathrm{km}^{2}\right)$ se estableció como el producto entre la distancia rastreada y la abertura de punta de alas. El primer término se calculó mediante la expresión pitagórica corregida por latitud, entre el inicio y término del arrastre, mientras que el segundo se estimó por método directo, basado en el empleo de instrumental electrónico denominado Trawlmaster (Notus Electronics Ltda.), equipado con un sensor sónico sumergido que mide la separación entre la punta de las alas.

Como índice de densidad se empleó la captura por unidad de área (CPUA), medido en kilos por kilómetro cuadrado $\left(\mathrm{kg} / \mathrm{km}^{2}\right)$, que es concordante con estudios realizados anteriormente por Arredondo \& Arana (1995), Acuña et al. (1997), Roa et al. (1999) y Escuela de Ciencias del Mar (2000a, 2000b). Para describir en términos geográficos éste índice, se calculó su media tanto para los lances to- tales como para aquellos con captura de $H$. diomedeae, del mismo modo, se contrastó respecto a la profundidad media de cada lance de pesca. Para obtener estimadores de índices de abundancia representativos de un determinado sector, se consideró el estimador de razón (Cochran, 1977), media aritmética y media generada con grupos creados en forma aleatoria. La variabilidad asociada a dichos estimadores se expresó mediante la construcción de intervalos de confianza utilizando el método del percentil (Efron \& Tibshirani, 1986; Manly, 1997).

La biomasa vulnerable $\left(\mathrm{B}_{\mathrm{f}}\right)$ presente en una unidad espacial (región o foco de abundancia) cualquiera, quedó determinada por el estimador de captura por unidad de área $C P U A_{f}$, amplificado o expandido al área total de la región o conglomerado $\left(\mathrm{A}_{\mathrm{f}}\right)$, de acuerdo a la siguiente expresión:

$$
B_{f}=C P U A_{f} \bullet A_{f}
$$

La desagregación del efectivo por sexos se efectuó mediante la siguiente formulación:

$$
B_{k}^{\text {sexo }}=B_{k}^{\text {total }} \cdot \frac{Y_{k}^{\text {sexo }}}{Y_{k}^{\text {total }}}
$$

donde $Y_{k}$ es la captura en peso lograda con la operación de la nave en el k-ésimo foco de abundancia. Posteriormente, la estratificación de esta biomasa en los distintos rangos de tallas $(L)$ se realizó con la siguiente expresión:

$$
B_{k, L}^{\text {sexo }}=B_{k}^{\text {sexo }} \cdot \frac{n_{k . L}{ }^{\text {sexo }} \cdot w_{L}^{\text {sexo }}}{Y_{k}^{\text {total }}}
$$

donde $n$ es la frecuencia numérica del L-ésimo estrato de tallas, $\mathrm{y} w_{L}$ el peso a la talla establecido con la relación talla-peso.

Adicionalmente, se registró la captura en peso (kg) de la fauna acompañante en los cruceros de pesca de prospección. La importancia relativa de las principales especies (\%RS), de acuerdo a la profundidad, se estableció como la relación entre su captura y la captura de gamba. 


\section{RESULTADOS}

Durante la prospección se realizaron 265 lances entre el 8 de agosto y el 16 de septiembre de 2002. Por razones operativas se asignó a cada nave un sector geográfico delimitado, de este modo, la nave "Elbe" operó sobre los fondos marinos del sector norte (32 $10^{\circ} \mathrm{S}$ a $\left.33^{\circ} 53^{\prime} \mathrm{S}\right)$, siendo posteriormente reemplazada por el pesquero "Don Stefan". Por otra parte, la nave "Crusoe I", prospectó el sector sur (335' $\mathrm{S}$ a $34^{\circ} 42^{\prime}$ 'S), embarcación que fue sustituida por el pesquero "Maori". La captura de $H$. diomedeae alcanzó 11,0 ton; de este total 4,1 ton se obtuvieron en el sector norte y 6,9 ton en el sector sur.

A fin de disponer de distribuciones de frecuencias de tallas en cada arrastre, se midió la longitud cefalotorácica a 50.713 individuos. De esta cantidad, 13.897 ejemplares $(27,4 \%)$ correspondieron a machos y $36.816(72,6 \%)$ a hembras. Con el propósito de establecer la relación de talla-peso, se midió y pesó un total de 3.058 ejemplares. Adicionalmente, para determinar la talla de madurez física en cada sexo, se registró la longitud total y la longitud cefalotorácica a $1.502(47,5 \%)$ machos y 1.662 hembras $(52,5 \%)$.

La longitud cefalotorácica global (machos + hembras) estuvo comprendida entre 12,0 y 72,0 mm, con promedio de $30,6 \mathrm{~mm}$ y moda en $28,0 \mathrm{~mm}$. Las hembras tuvieron una longitud media de $32,0 \mathrm{~mm}$ (30,0 mm de moda) y los machos $27,1 \mathrm{~mm}(24,0$ $\mathrm{mm}$ de moda). En el sector norte la talla promedio global fue mayor, alcanzando una media de $31,8 \mathrm{~mm}$ (30,0 $\mathrm{mm}$ de longitud modal), mientras que en el sector sur fue de $29,5 \mathrm{~mm}(28,0 \mathrm{~mm}$ de longitud modal) (Fig. 2).

$\mathrm{El}$ análisis de las relaciones corporales $\mathrm{L}_{\mathrm{t}}-\mathrm{L}_{\mathrm{c}}$ indicó en machos un máximo en la función de verosimilitud a $32,5 \mathrm{~mm}$ de $\mathrm{L}_{\mathrm{c}}$, mientras que en las hembras, fue a los $36,5 \mathrm{~mm}$ de $\mathrm{L}_{\mathrm{c}}$. Contrastadas las hipótesis nulas de igualdad de rectas de regresión de individuos inmaduros y maduros en cada sexo, éstas fueron rechazadas (Fig. 3).

El análisis de la proporción sexual a la talla, evidenció el predominio de machos en el rango inferior, tendencia que se observó hasta los $25 \mathrm{~mm}$ de $\mathrm{L}_{\mathrm{c}}$, mientras que sobre esa talla se apreció una mayor proporción de hembras (Fig. 4). El análisis de la relación talla-peso indicó relaciones de tipo alométrico negativo en ambos sexos (Tabla 1).

La gamba se encontró a lo largo de toda el área de prospección. En profundidad, las capturas se registraron entre 330 y $682 \mathrm{~m}$, que corresponde a la profundidad máxima rastreada en esta oportunidad. De los 179 lances en los que se capturó gamba, sobre el $60 \%$ se concentró a profundidades mayores de $500 \mathrm{~m}$ (Fig. 5).

Considerando los lances con capturas positivas (330 a $682 \mathrm{~m}$ de profundidad), los rendimientos de pesca estuvieron comprendidos entre 0,01 y $540 \mathrm{~kg} /$ h.a., con un promedio global de $32,5 \mathrm{~kg} / \mathrm{h}$.a. Los sectores norte y sur presentaron valores medios de 36,2 y $29,3 \mathrm{~kg} / \mathrm{h}$. a., respectivamente.

Para evaluar la biomasa vulnerable, se utilizó la abertura media de punta de alas (APA) de las redes de las naves "Elbe" y "Crusoe I", que correspondió a 13,8 y $13,3 \mathrm{~m}$, respectivamente. La abundancia relativa media en los sectores norte y sur, considerando la totalidad de los lances efectuados, alcanzó 408 y $494 \mathrm{~kg} / \mathrm{km}^{2}$, respectivamente. Al considerar sólo aquellos lances con presencia de gamba (68\%), los estimados alcanzan a 557 y $751 \mathrm{~kg} / \mathrm{km}^{2}$, respectivamente. Al considerar la CPUA por rango de profundidad, no se detectaron diferencias importantes, dado que mostró un patrón de comportamiento prácticamente uniforme a partir de aproximadamente los $450 \mathrm{~m}$ (Fig. 6).

La distribución de los lances con y sin captura, delimitaron dos grandes unidades espaciales con presencia de gamba, dentro de los cuales se efectuaron 59 y 62 lances de pesca, respectivamente. Ambos conglomerados estuvieron separados por el cañón submarino de San Antonio, y se extendieron sobre el fondo abarcando superficies respectivamente de $620,6 \mathrm{~km}^{2}$ en el caladero norte (desde punta Hueso a rada Algarrobo) y de $539,2 \mathrm{~km}^{2}$ en el caladero sur (entre San Antonio y punta Sirena) (Fig. 7).

$\mathrm{Al}$ interior de estos caladeros la CPUA promedio (media aritmética) se estimó en 574 y $722 \mathrm{~kg} /$ $\mathrm{km}^{2}$, respectivamente. Las biomasas vulnerables estuvieron comprendidas entre 343 y 360 ton en el caladero norte y entre 360 y 390 ton en el caladero sur, con un estimado global entre 702 y 745 ton (Tabla 2). Así, tomando en cuenta la biomasa vulnerable determinada mediante el estimador media aritmética y el aporte en peso por sexo, la fracción disponible en cada caladero por sobre la talla media de madurez respectiva de cada sexo, correspondió a 34,5 ton en machos y 203,2 ton en hembras, con un total de 237,7 ton en toda el área prospectada.

De acuerdo al índice $\mathrm{RS} \%$, las principales espe- 


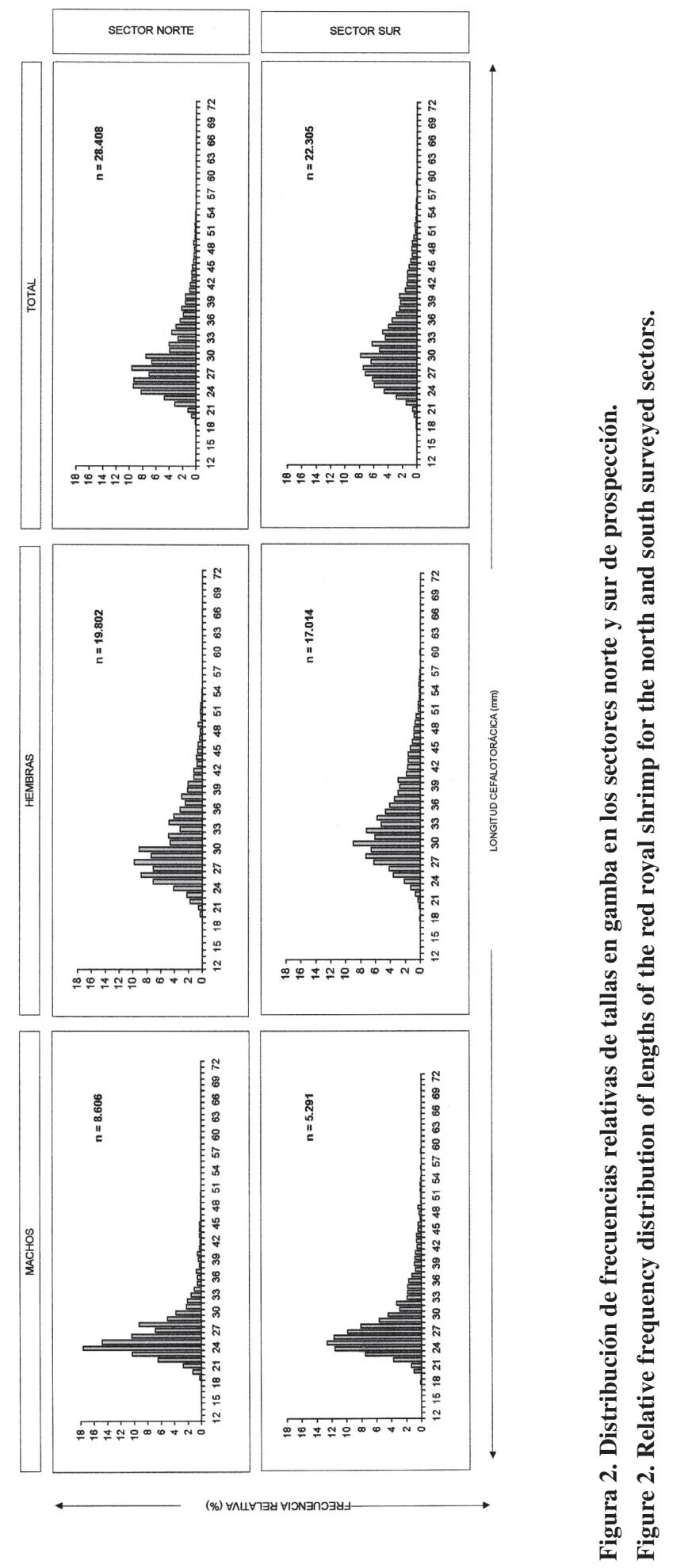


Tabla 1. Parámetros regresionales de la relación talla-peso (relación de poder) de la gamba (agosto-septiembre de 2002).

Table 1 Regression parameters for the length-weight relationship (power relationship) of the red royal shrimp (August-September 2002).

\begin{tabular}{|c|c|c|c|c|c|c|}
\hline \multirow[b]{2}{*}{ Parámetro } & \multicolumn{3}{|c|}{$\begin{array}{c}\text { Sector norte } \\
\left(32^{\circ} 10-33^{\circ} 53 ' S\right)\end{array}$} & \multicolumn{3}{|c|}{$\begin{array}{c}\text { Sector sur } \\
\left(33^{\circ} 53-34^{\circ} 42^{\prime} S\right)\end{array}$} \\
\hline & Machos & Hembras & Total & Machos & Hembras & Total \\
\hline a & 0,0023 & 0,0029 & 0,0032 & 0,0045 & 0,0026 & 0,0042 \\
\hline $\mathrm{b}$ & 2,4633 & 2,3865 & 2,3636 & 2,2790 & 2,4204 & 2,2975 \\
\hline $\mathrm{n}$ & 846 & 734 & 1.580 & 883 & 595 & 1.478 \\
\hline SCE & 9,37 & 6,54 & 16,50 & 8,64 & 4,88 & 14,20 \\
\hline Coeficiente de correlación (R) & 0,96 & 0,98 & 0,98 & 0,97 & 0,98 & 0,98 \\
\hline
\end{tabular}
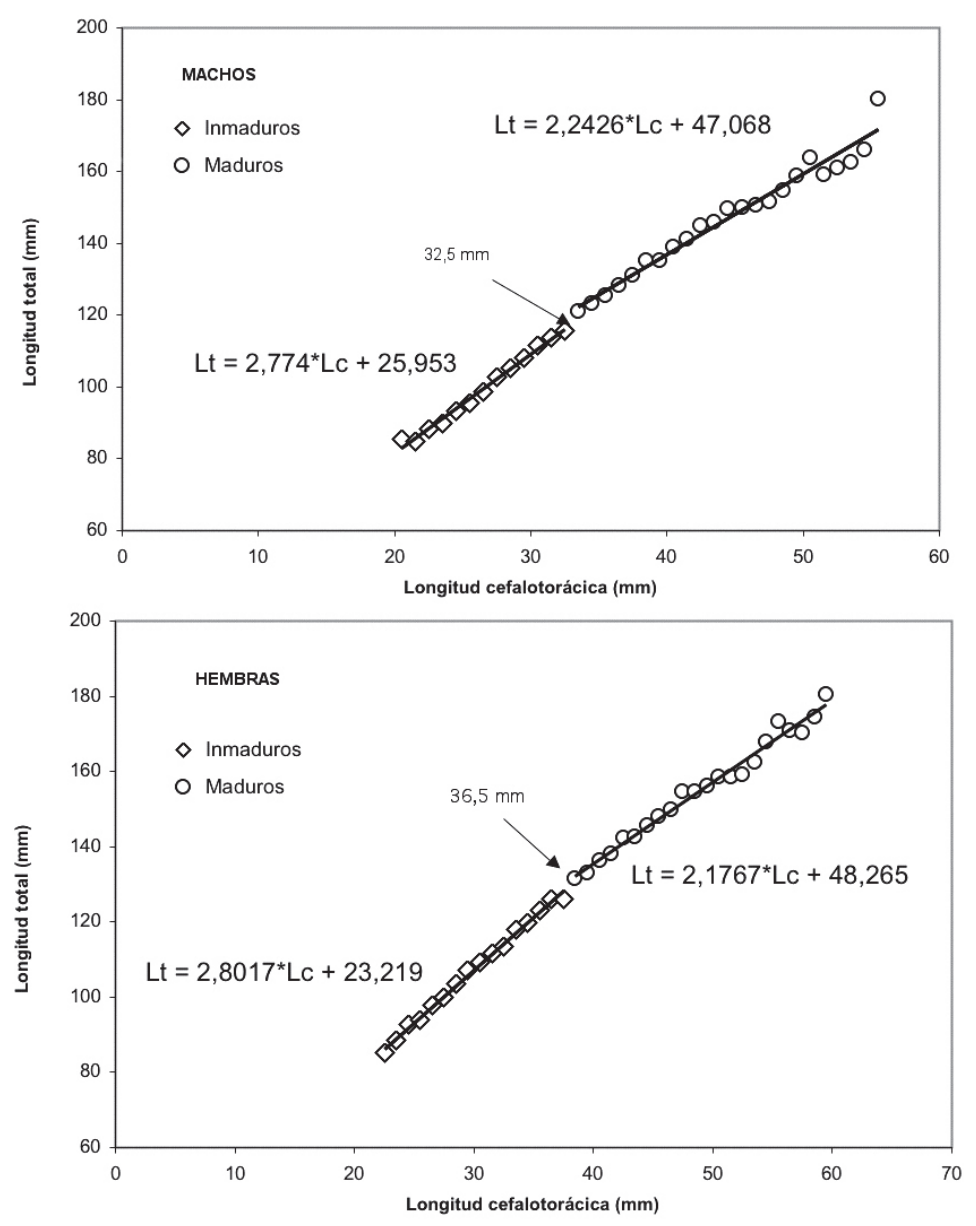

Figura 3. Relación entre la longitud cefalotorácica $\left(L_{c}\right)$ y la longitud total $\left(L_{t}\right)$ en la gamba. Las flechas indican cambio en la relación biométrica, que correspondería a la talla en que esta especie alcanza la madurez física.

Figure 3. Relationship between carapace $\left(L_{c}\right)$ and total length $\left(L_{t}\right)$ of the red royal shrimp. Arrows indicate the shift in the biometric relationship corresponding to the size at which the species reaches its physical maturity. 


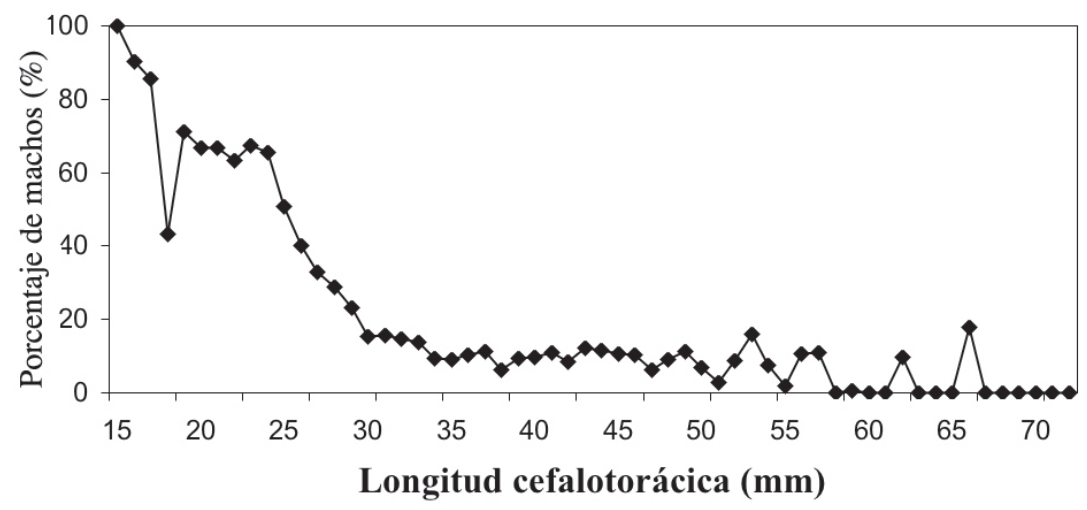

Figura 4. Proporción sexual a la talla en la gamba.

Figure 4. Sex proportion in relation to the carapace length in red royal shrimp.

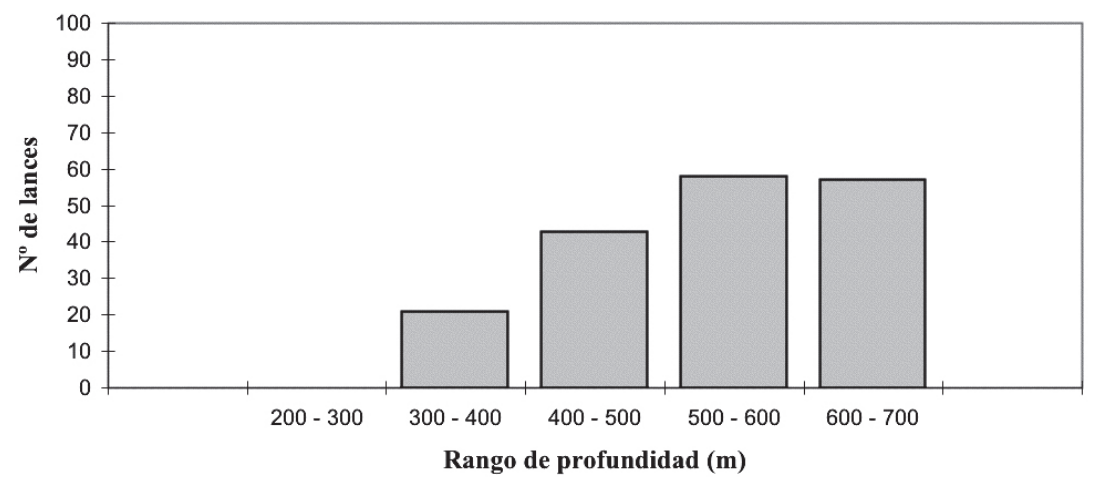

Figura 5. Número de lances con captura de gamba, por rango de profundidad.

Figure 5. Number of hauls with red royal shrimp, by surveyed depth ranges.

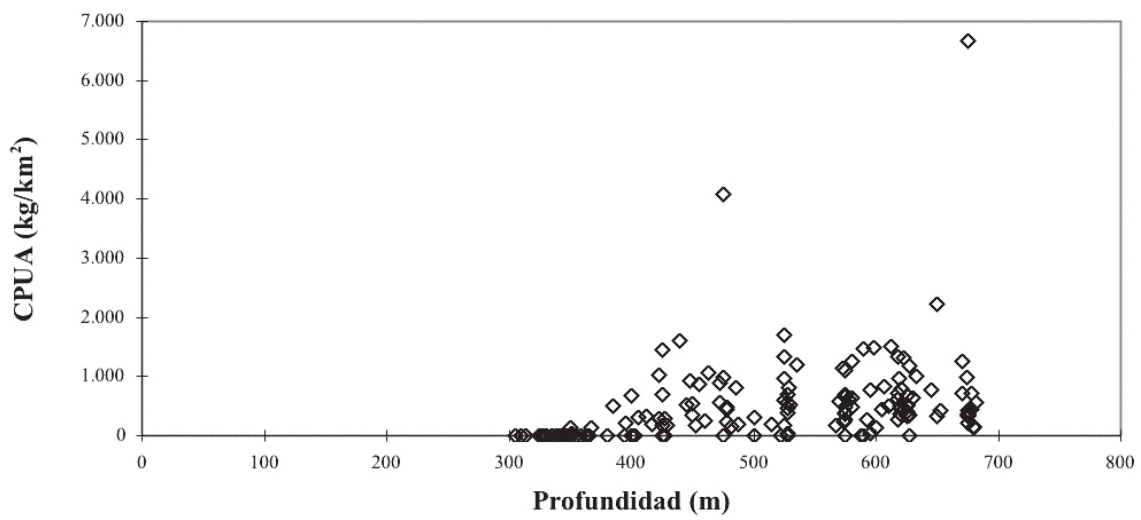

Figura 6. Captura por unidad de área (CPUA) de gamba, en relación a la profundidad.

Figure 6. Catch per unit of area (CPUA) of red royal shrimp by depth. 


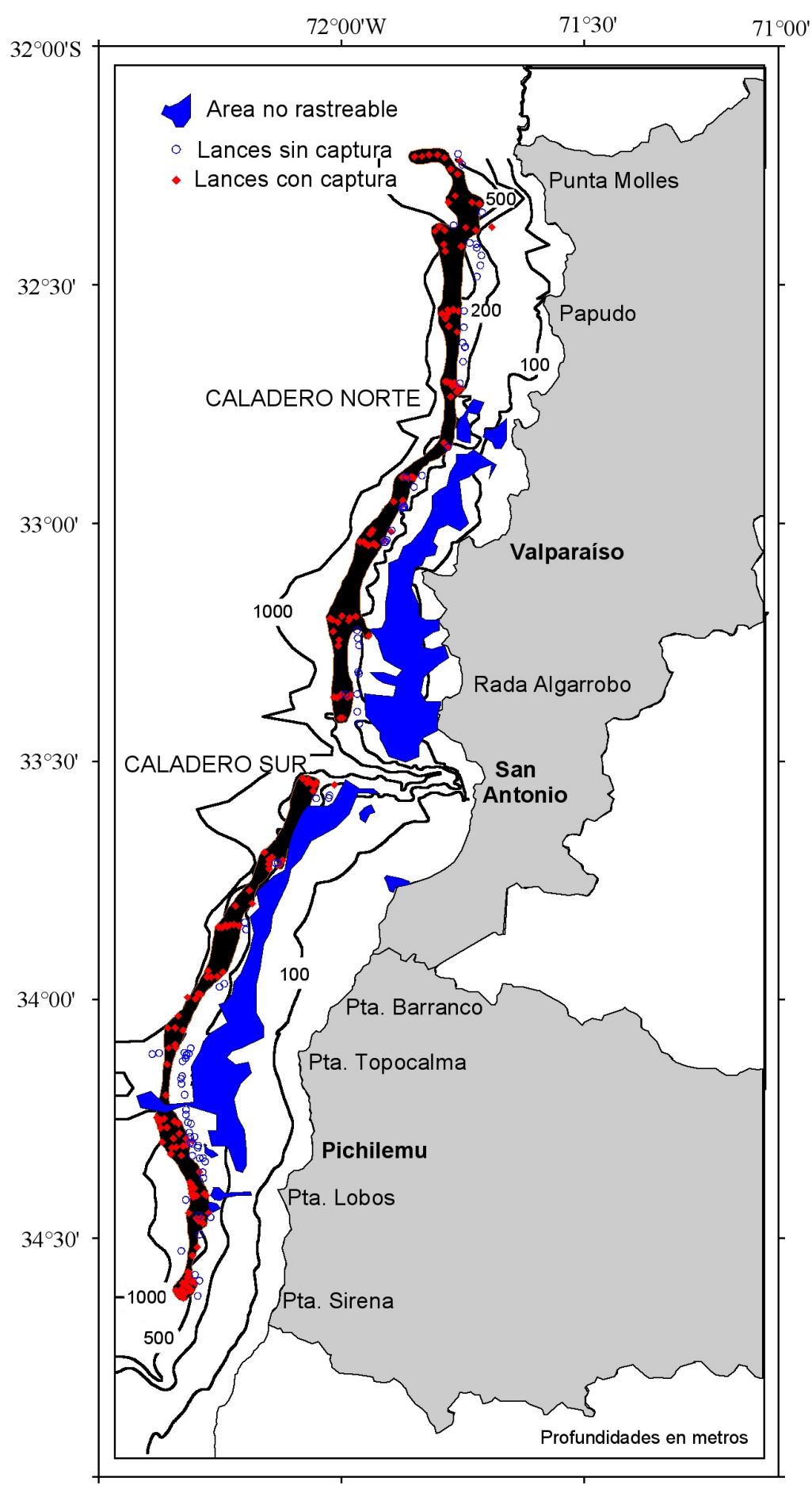

Figura 7. Lances con captura (rojo), sin captura (azul) y caladeros (achurado) de gamba en el crucero de prospección.

Figure 7. Red royal shrimp hauls with catch (red), without catch (blue), and fishing grounds (stippled) in the survey cruise. 
cies comerciales capturadas junto a $H$. diomedeae fueron camarón nailon (Heterocarpus reedi) $(0,1 \%$ a $103,1 \%)$, merluza común (Merluccius gayi) $(0,1 \%$ a $22,2 \%$ ), langostino amarillo (Cervimunida johni) $(0,0 \%$ a $2,1 \%)$ y congrio dorado (Genypterus blacodes $)(0,3 \%$ a 39,2\%), siendo el pejerrata (Coelorhynchus aconcagua) la especie que presentó la mayor captura asociada $(6,9 \%$ a $972,6 \%)$. No obstante, es necesario destacar que la composición de la fauna asociada presentó notoria variación de acuerdo al rango de profundidad en que se efectuaron los arrastres. Así, a partir de los 600 m de pro- fundidad la pesca de gamba presenta como fauna acompañante $C$. aconcagua $(\mathrm{RS} \%=6,9 \%)$ y cantidades marginales de otras especies.

En el caso particular de camarón nailon, que es actualmente el principal crustáceo explotado en el talud continental, sus capturas se concentraron mayoritariamente a profundidades inferiores a 450 m. De acuerdo con ello, se determinó que al aumentar la profundidad disminuyó progresivamente el porcentaje relativo de esta especie respecto a la captura de la gamba (Tabla 3).

Tabla 2. Número de lances de pesca utilizados en la evaluación, CPUA y biomasa vulnerable de gamba por caladero. MA: Media aritmética, ER: Estimador de razón, GA: Media de grupos aleatorios.

Table 2. Number of fishing hauls used in the assessment, CPUA and vulnerable biomass, by fishing ground. MA: Mean average, ER: Ratio index, GA: Random mean groups.

\begin{tabular}{|c|c|c|c|c|}
\hline \multirow[t]{2}{*}{ Caladero } & \multirow[t]{2}{*}{ Localización } & \multicolumn{2}{|c|}{ Lances utilizados en la evaluación } & \multirow[t]{2}{*}{ Area $\left(\mathbf{k m}^{2}\right)$} \\
\hline & & $\begin{array}{c}\text { Lances en el } \\
\text { caladero }\end{array}$ & $\begin{array}{c}\text { Lances } \\
\text { con captura }\end{array}$ & \\
\hline Norte & $32^{\circ} 13^{\prime} \mathrm{S}-33^{\circ} 25^{\prime} \mathrm{S}$ & 59 & 56 & 620,6 \\
\hline Sur & $33^{\circ} 31^{\prime} \mathrm{S}-33^{\circ} 38^{\prime} \mathrm{S}$ & 62 & 61 & 539,2 \\
\hline Totales & & 121 & 117 & $1.159,8$ \\
\hline
\end{tabular}

\begin{tabular}{|c|c|c|c|c|c|c|c|c|c|}
\hline \multirow[t]{2}{*}{ Caladero } & \multicolumn{3}{|c|}{$\begin{array}{c}\text { CPUA } \\
\left(\mathbf{k g} / \mathbf{k m}^{2}\right)\end{array}$} & \multicolumn{2}{|c|}{ Biomasa (ton) MA } & \multicolumn{2}{|c|}{ Biomasa (ton) ER } & \multicolumn{2}{|c|}{ Biomasa (ton) GA } \\
\hline & MA & ER & GA & Valor & Intervalo & Valor & Intervalo & Valor & Intervalo \\
\hline Norte & 574,1 & 552,3 & 579,5 & 356,3 & $278,93-435,4$ & 342,7 & $278,4-405,3$ & 359,6 & $259,2-565,9$ \\
\hline \multirow[t]{2}{*}{ Sur } & 722,4 & 666,3 & 714 & 389,5 & $310,08-484,3$ & 359,3 & $301,2-427,6$ & 384,3 & $298,7-578,3$ \\
\hline & \multicolumn{3}{|c|}{ Totales } & 745,8 & $644,8-846,8$ & 702 & $606,4-797,5$ & 743,9 & $743,9-536,0$ \\
\hline
\end{tabular}

Tabla 3. Captura relativa (RS\%), por especie y rango de profundidad respecto a la captura de gamba.

Table 3. Relative catch (RS\%), by species and depth range in relation to catch of the red royal shrimp.

\begin{tabular}{|c|c|c|c|c|c|}
\hline \multirow[b]{2}{*}{$\begin{array}{c}\text { Rango de } \\
\text { profundidad (m) }\end{array}$} & \multicolumn{5}{|c|}{ Especie } \\
\hline & $\begin{array}{l}\text { Heterocarpus } \\
\text { reedi }\end{array}$ & $\begin{array}{c}\text { Merluccius } \\
\text { gayi }\end{array}$ & $\begin{array}{c}\text { Genypterus } \\
\text { blacodes }\end{array}$ & $\begin{array}{c}\text { Coelorhynchus } \\
\text { aconcagua }\end{array}$ & $\begin{array}{c}\text { Cervimunida } \\
\text { johni }\end{array}$ \\
\hline $301-400$ & 226,0 & 5,1 & 0,8 & 87,3 & 4,9 \\
\hline $401-500$ & 103,1 & 22,2 & 39,2 & 972,6 & - \\
\hline $501-600$ & 4,2 & 0,8 & 0,2 & 115,0 & 2,1 \\
\hline $601-700$ & 0,1 & 0,1 & 0,3 & 6,9 & - \\
\hline
\end{tabular}




\section{DISCUSIÓN}

Las capturas de $H$. diomedeae se obtuvieron prácticamente a lo largo de toda el área estudiada, lo cual indica que se distribuye sobre el fondo marino de manera similar al camarón nailon, es decir, en forma continua sobre el talud continental en orientación norte-sur. Al parecer esta especie no presenta agregaciones del tipo "focos de abundancia" como se han determinado en el langostino amarillo $(C$. johni) y langostino colorado (Pleuroncodes monodon) (Escuela de Ciencias del Mar, 2000a; Barbieri et al., 2000). Pese a ello, en el presente trabajo se consideraron dos unidades espaciales distintas para evaluar la biomasa vulnerable (caladeros norte y sur), por cuanto las características topográficas en el sector de los 33³0’S (cañón submarino de San Antonio), hicieron imposible la realización de faenas de pesca en ese lugar, interrumpiendo la continuidad en la distribución del recurso.

El rango batimétrico en el cual se localizó la gamba (330-682 m), es concordante con lo indicado por Noziglia \& Arana (1976) e idéntico en su rango inferior al señalado por Yáñez (1974) entre los 32³0's y 3330'S (325-1.000 m). Al respecto, la presencia de este crustáceo en estas profundidades estaría en relación con las características físicas de la masa de Agua Intermedia Antártica, cuya temperatura fluctúa entre 7,5 y $3,5^{\circ} \mathrm{C}$, existiendo una relación inversa entre la abundancia de esta especie y la intensificación de esa masa de agua en el Pacífico suroriental (Noziglia \& Arana, 1976).

De acuerdo a la estructura de tallas obtenida, se determinó en la gamba una longitud cefalotorácica media de 30,6 mm, siendo las hembras de tamaño notoriamente mayor que los machos (promedio de 32,0 y $27,1 \mathrm{~mm}$, respectivamente). Respecto de las tallas modales, dichos valores se situaron en 24 y $30 \mathrm{~mm}$ correspondientemente en machos y hembras. En este sentido, se debe indicar que tales modas son inferiores a las registradas por Noziglia \& Arana (1976), quienes indicaron modas de 29 y $33 \mathrm{~mm}$ en machos y 35 y 40 mm en hembras, en julio-noviembre de 1973 y 1974, respectivamente, en una zona similar a la prospectada en esta oportunidad, ubicada entre Papudo (3230'S) y Punta Talca (33⒉ $\left.5^{\prime} \mathrm{S}\right)$.

$\mathrm{Al}$ respecto, en aguas peruanas se han indicado $\mathrm{L}_{\mathrm{c}}$ entre 20 y $66 \mathrm{~mm}$, y tallas promedio de 28,4 en machos y 36,4 mm en hembras (Vélez et al., 1992). De modo similar, en aguas chilenas de la zona centro-sur (3800'S-42³0'S), Leiva et al. (1997) re- gistraron tallas comprendidas entre 22 y $66 \mathrm{~mm}$ de $\mathrm{L}_{\mathrm{c}}$. De este modo, ambos estudios presentan un rango más acotado al obtenido en la presente prospección (12 a $72 \mathrm{~mm}$ ), pero con promedios de tallas mayores en aguas peruanas a los establecidos en la presente investigación (27,1 y 32,0 mm en machos y hembras, respectivamente).

Otro aspecto relevante es que en las capturas, la cantidad de hembras fue significativamente superior a la de machos, y éstas se distribuyeron en un rango de talla notoriamente superior (Fig. 4). Estos resultados son coincidentes con los determinados previamente por Noziglia \& Arana (1976); Gaete \& Arana (1986); Vélez et al. (1992) y Leiva et al. (1997).

El análisis efectuado por separado en los sectores norte y sur, indica mayores longitudes promedios y modales en este último. Esto es concordante con la información proporcionada por capitanes de pesca (Bustos \& Arana, 2003), que señalan su preferencia por realizar faenas extractivas en caladeros localizados en el sector sur, debido a las mayores tallas allí obtenidas.

Las hembras de Penaeidae no portan los huevos, sino que los liberan al medio, donde completan su desarrollo embrionario. De allí que el establecimiento de la talla de primera madurez se deba realizar mediante otros procedimientos como es la madurez física o funcional, que corresponde a la longitud a la que los organismos varían el crecimiento relativo de algunas estructuras, que está relacionado con la maduración de sus órganos sexuales y morfología requerida para lograr un apareamiento exitoso (George \& Morgan, 1979; Grey, 1979; Conan \& Comeau, 1986).

Al igual que en Noziglia \& Arana (1976), en las hembras la madurez física se alcanzó a $36,5 \mathrm{~mm}$, en cambio en los machos, se determinó a 32,5 mm, tamaño levemente mayor a lo estimado por estos autores $(30,0 \mathrm{~mm})$. De acuerdo a la distribución de tallas, los resultados obtenidos implican que actualmente la flota vulnera principalmente ejemplares inmaduros, por cuanto el $94,4 \%$ y $92,6 \%$ de $\operatorname{los}$ machos, y el $85,3 \%$ y $77,8 \%$ de las hembras, estarían bajo dicha talla de madurez física en los sectores norte y sur, respectivamente, peligrando así la sustentabilidad de su explotación.

En este sentido, se observó que la metodología empleada requiere de un alto nivel de precisión en la medición de tallas a fin de asegurar robustez en los estimados. A partir del análisis de los datos rela- 
tivos a machos, se detectó igualmente un máximo a los 26,5 $\mathrm{mm}$ de $\mathrm{L}_{\mathrm{c}}$, talla inferior a la calculada por Noziglia \& Arana (1976), y que sin embargo, corresponde al percentil 70 y 60 de la distribución de frecuencias de tallas en las áreas norte y sur, respectivamente. De acuerdo a esto, es conveniente efectuar análisis histológico de las gónadas de $H$. diomedeae a fin de dilucidar con certeza la talla de primera madurez sexual. Al respecto, cabe indicar que Vélez et al. (1992) tuvieron dificultad en visualizar los estadios de madurez al realizar análisis macroscópico de las gónadas.

Respecto de la abundancia relativa de la gamba y considerando sólo aquellos lances positivos (68\%), se calcularon valores promedios de 557 y $751 \mathrm{~kg}$ / $\mathrm{km}^{2}$ en los sectores norte y sur, respectivamente. Con relación a la biomasa vulnerable, los antecedentes disponibles son escasos, ya que la única evaluación previa de esta especie fue realizada por Yáñez \& Barbieri (1974), quienes estimaron en invierno de 1973 un total de 195,2 ton entre Papudo y Punta

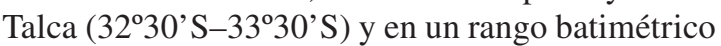
comprendido entre 200 y $600 \mathrm{~m}$, intervalo similar al utilizado en el presente trabajo (caladero norte), aunque en esta oportunidad se trabajó en un área de mayor extensión latitudinal.

Las capturas acumuladas de gamba entre enero y septiembre de 2002 alcanzó al menos 226 ton (A. Quintanilla, com. pers.). De este modo, si se considera la cantidad desembarcada en los meses previos a la evaluación, es posible proyectar la biomasa vulnerable al $1^{\circ}$ de enero de 2002 a un valor entorno a 1.000 ton.

En aguas peruanas Vélez et al. (1992) obtuvieron rendimientos promedio de $56 \mathrm{~kg} / \mathrm{h}$.a. entre 540 y 880 m en los alrededores del Banco de Máncora (norte de Perú), con una red de 13,5 m de abertura horizontal, similar a la empleada en el presente estudio. En el presente trabajo, los datos recopilados, expresados en términos de CPUE (kg/h.a.), muestran un promedio inferior, de $32,5 \mathrm{~kg} / \mathrm{h} . \mathrm{a}$. $(36,2 \mathrm{y}$ $29,3 \mathrm{~kg} / \mathrm{h}$.a. en los sectores norte y sur, respectivamente). Este antecedente, y las CPUA registradas por Leiva et al. (1997), no superiores a $14 \mathrm{~kg} / \mathrm{km}^{2}$ en los 42³0'S (entre 408 y $494 \mathrm{~kg} / \mathrm{km}^{2}$ en el presente trabajo), serían consistentes con el supuesto de una menor densidad de esta especie hacia el sur de su área de distribución (Noziglia \& Arana, 1976).

Al comparar los rendimientos batimétricamente, los datos recopilados en Perú por Vélez et al. (1992) señalan valores de CPUE (kg/h.a.) uniformes entre
600 y $700 \mathrm{~m}$, con un incremento a profundidades mayores de $725 \mathrm{~m}$, sugiriendo así una mayor disponibilidad en aguas más profundas. Por otra parte, Leiva et al. (1997) indican que las mayores CPUA se concentran entre los 600 y $700 \mathrm{~m}$ de profundidad $\left(10 \mathrm{~kg} / \mathrm{km}^{2}\right)$. En el presente trabajo, los datos recopilados hasta aproximadamente $105700 \mathrm{~m}$ no muestran patrones que indiquen que la CPUA se relacione con la profundidad. No obstante, como no se efectuaron lances de pesca a más de 700 m, no es factible constatar un posible aumento de los rendimientos en aguas más profundas.

Considerando los resultados obtenidos, se puede señalar que los rendimientos de pesca gamba son menores que los reportados para otras especies de crustáceos actualmente explotados en aguas chilenas. De acuerdo a los niveles de biomasa vulnerable estimados, este recurso no permitiría el desarrollo de actividades extractivas a gran escala, siendo más factible que las faenas de pesca dirigidas a su captura constituyan actividades complementarias a la explotación de otros recursos como $H$. reedi, $P$. monodon y $C$. johni, especialmente durante los períodos en que éstos se encuentran en veda.

\section{AGRADECIMIENTOS}

Se agradece a la Subsecretaría de Pesca por la autorización de esta pesca de investigación y, en forma particular, al Sr. Italo Campodonico G. por su apoyo. Así también, al personal de Pesquera Quintero S.A., en especial, al Sr. Mario Morozin B. (Gerente General), al Sr. Andrés Quintanilla G. (Jefe de Producción), al Sr. José Bastidas R. (Jefe de Flota), y a los capitanes y tripulantes de las naves que participaron en la prospección.

\section{REFERENCIAS}

Acuña, E., H. Arancibia, A. Mujica, L. Cid \& R. Roa. 1997. Análisis de la pesquería y evaluación indirecta del stock de langostino amarillo en la III y IV Regiones. Informe Final, Proyecto FIP 9608. Fondo de Investigación Pesquera, 153 pp.

Alverson, D. \& W. Pereyra. 1969. A study of demersal fishes and fisheries of the northeastern Pacific Ocean. An evaluation of exploratory fishing methods and analytical approaches to stock size and yield forecast. J. Fish. Res. Bd. Can., 26: 1985 2001 . 
Arana, P., M. Ahumada \& A. Guerrero. 2003. Evaluación directa de camarón nailon entre la II y VIII Regiones, año 2002 (Proyecto FIP N²002-05). Informe Final. Estud. Doc., Univ. Católica Valparaíso, 05/2003: 318 pp.

Arana, P. \& A. Cristi. 1971. Parámetros biométricos de la gamba (Hymenopenaeus diomedeae). Inv. Mar., 2(2): 21-40.

Arredondo, M. \& P. Arana. 1995. Evaluación directa del stock de langostino amarillo (Cervimunida johni) entre Valparaíso (V Región) y Talcahuano (VIII Región). En: P. Arana (ed). Evaluación directa del stock de langostino amarillo y estado de situación del recurso entre la V y VIII Región. Estud. Doc., Univ. Católica Valparaíso, 48/95: 231281.

Barbieri, M.A., C. Canales, B. Leiva, R. Bahamonde, T. Peñailillo, H. Pool, C. Montenegro, S. Lillo, P. Gálvez, G. Muñoz \& C. Vicencio. 2000. Evaluación directa de langostino colorado de la I a IV Regiones, 1999. Informe Final, Proyecto FIP 99-30. Instituto de Fomento Pesquero, 96 pp.

Bustos, R. \& P. Arana. 2003. Pesquería de camarón nailon (Heterocarpus reedi) y gamba (Haliporoides diomedeae) desarrollada por Pesquera Quintero S.A. en las Regiones IV, V y VI: enero-marzo, 2003. Informe Técnico $N^{\circ} 1 / 2003$ : 68 pp.

Canales, C., M.A. Barbieri, R. Bahamonde, B. Leiva, P. Arana, S. Palma \& T. Melo. 2001. Evaluación directa de langostino colorado y langostino amarillo entre la II y VIII Región, año 2001. Informe Final, Proyecto FIP 2001-06. Instituto de Fomento Pesquero, 202 pp.

Cochran, W. 1977. Sampling techniques. John Wiley \& Sons, New York, 428 pp.

Conan, G.Y. \& M. Comeau. 1986. Functional maturity and terminal molt of male snow crab, Chionoecetes opilio. Can. J. Fish. Aquat. Sci., 43: 1710-1719.

Dixon, W. \& J. Masey. 1957. Introduction to statistical analysis. McGraw-Hill, New York, 488 pp.

Efron, B. \& R. Tibshirani. 1986. Bootstrap methods for standard errors, confidence intervals, and other measures of statistical accuracy. Statistical Science, 2(1): 54-77.
Escuela de Ciencias del Mar. 2000a. Evaluación directa de camarón nailon, langostino amarillo y langostino colorado, año 2000. Informe Final Proyecto FIP 2000-05. Estud. Doc., Univ. Católica Valparaíso, 21/2000: 315 pp.

Escuela de Ciencias del Mar. 2000b. Evaluación directa de langostino colorado, en la Región centrosur de Chile, 2000. Informe Final. Estud. Doc., Univ. Católica Valparaíso, 20/2000: 142 pp.

Faxon, W. 1895. Reports on an exploration off west coast of Mexico, Central and South America, and off Galapagos islands, in charge of Alexander Agassiz, by the U.S. Fish Commission steamer Albatros. XV The stalk-eyed Crustacea. Mem. Mus. Comp. Zool., Harv. Coll., 18: 1-292.

Gaete, V. \& P. Arana. 1986. Proporción sexual en tres especies de camarones explotadas frente a la costa central de Chile. Invest. Mar., Valparaíso, 14: 4568.

George, R.W. \& G.R. Morgan. 1979. Linear growth stages in the rock lobster (Panulirus versicolor) as a method for determining size at first physical maturity. Rapp. P.-v. Réun. Cons. int. Explor. Mer, 175: 182-185.

Grey, K.A. 1979. Estimates of size of first maturity of the western rock lobster, Panulirus cygnus, using secondary sexual characteristics. J. Mar. Freshw. Res., 30: 785-791.

Gray, E.H. \& C.L. Newcombe. 1938. The relative growth of parts in the blue crab Callinectes sapidus Rathbun. Growth, 2(3): 235-246.

Guerrero, A., R. Bustos, M. Ahumada \& P. Arana. 2003. Monitoreo de las pesquerías de crustáceos bentónicos desarrolladas entre la III y IV Región, año 2001. Informe Convenio Marco, Nº/2003: 236 pp.

Henríquez, G., L. Rodríguez \& I. Kong. 1981. Exploración y prospección de recursos pesqueros del talud continental. Informe Técnico. Santiago, Chile, IFOP (AP 81-5): 62 pp.

Illanes, J.E. \& O. Zúñiga. 1972. Contribución a la morfología de la "gamba" (Hymenopenaeus diomedeae, Faxon) de la zona central de Chile (Crustacea: Decapoda: Penaeidae). Invest. Mar., Valparaíso, 3(1): 1-22. 
Inostroza, M.C. 1981. Regresión lineal a varias fases. Tesis de Matemáticas, Instituto de Matemáticas, Univ. Católica Valparaíso, 112 pp.

Leiva, B., R. Bahamonde, M. Leiva, M. Donoso, A. Irarrázabal, M.I. Ortego \& S. Pino. 1997. Prospección de recursos demersales en aguas exteriores de las Regiones X y XI. Informe Final, Proyecto FIP 95-19. Instituto de Fomento Pesquero, 113 pp.

Manly, B. 1997. Randomization, bootstrap and montecarlo methods in biology. Chapman \& Hall, London, 398 pp.

Neter, J. \& W. Wasserman. 1974. Applied linear statistical models. Regresion, analysis of variance, and experimental designs. Edit. Richard D. Irwin, Ontario, 842 pp.

Noziglia, L. \& P. Arana. 1976. Observaciones biológico-pesqueras de la gamba, Hymenopeneaeus diomedeae Faxon 1893 en el Pacífico Suroriental (Crustacea: Decapoda: Penaeidae). Rev. Com. Perm. Pacífico Sur, 5: 63-83.

Pérez-Farfante, I. 1977. American solenocerid shrimps of the genera Hymenopenaeus, Haliporoides, Pleoticus, Hadropeneaus new genus and Masapeneaus new genus. US Fish. Bull., 75: $261 \mathrm{pp}$.

Quandt, R.E. 1958. The estimation of the parameters of a linear regression system obeying two separate regime. J.A.S.A., 53: 873-880.

Reigada, A.L., M. Negreiros-Fransozo \& F. Mantelatto. 1994. Appraisal of the cheliped's size on Hepatus pudibundus (Herbst, 1785) (Crustacea, Calappidae) in function of sex and maduration. Arq. Biol. Tecnol., 37(4): 797-807.

Recibido: 23 junio 2003; Aceptado: 30 octubre 2003
Roa, R., F. Tapia, L. Cid, M. Lépez, C. Díaz \& H. Arancibia. 1999. Evaluación directa de camarón nailon entre la II y VIII Regiones. Informe Final, FIP 98-03. Universidad de Concepción, 115 pp.

Somerton, D.A. 1980. A computer technique for estimating the size of sexual maturity in crabs. Can. J. Fish. Aquat. Sci., 37: 1488-1494.

Sparre, P. \& S.C. Venema. 1995. Introducción a la evaluación de recursos pesqueros tropicales. Parte 1. Manual. FAO Fish. Tech. Paper, N³06.1, Rev. 2, Roma, 420 pp.

Troadec, J.P. 1980. Utilization of resource survey results in stock assessment. En: Selected lectures from the CIDA/FAO/CECAF seminar on fishery resource evaluation. Casablanca, Morocco, 6-24 March 1978. Rome, FAO, Canada Funds-in-Trust, FAO/TF/INT 180(c) Suppl.: 139-152.

Vélez, J., A. Kameya, C. Yamashiro, N. Lostaunau \& O. Valiente. 1992. Investigación del recurso potencial langostino rojo de profundidad a bordo del BIC "Fridtjof Nansen" (25 de abril-25 de mayo, 1990). Informe Inst. Mar Perú-Callao, 104: 24 pp.

Yáñez, E. 1974. Distribución y abundancia relativa estacional de los recursos disponibles a un arte de arrastre camaronero frente a la costa de Valparaíso (invierno-primavera 1972). Invest. Mar., Valparaíso, 5(5): 125-138.

Yáñez, E. \& M.A. Barbieri. 1974. Distribución y abundancia relativa de los recursos disponibles a un arte de arrastre camaronero frente a la costa de Valparaíso (invierno 1973). Invest. Mar., Valparaíso, 5(6): 137-156. 